\title{
Digital technologies for organizing the intelligent learning environment
}

\author{
A I Dashkina ${ }^{1}, A M$ Kobicheva $^{1, *}, M$ Odinokaya $^{1}$, and $D A$ Tarkhov $^{1}$ \\ ${ }^{1}$ Peter the Great St.Petersburg Polytechnic University, 29, Polytechnicheskaya str., Saint-Petersburg, \\ Russia
}

\begin{abstract}
This article describes the problem of computer simulation of the educational process at a research university. We consider some models and methods of organizing the intelligent learning environment described by a number of researchers. We also come up with a few ideas of creating an intelligent learning environment and organizing the learning process, such as a hierarchical educational system, the model of which was tested in our previous studies.
\end{abstract}

\section{Introduction}

The development of information technologies along with the shift to a competence educational paradigm has brought about drastic changes in the forms and methods of organizing learning environments. In the intelligent learning environment, learners set individual educational goals that can be achieved independently on the basis of their previous knowledge and existing practical skills by setting the stage for hypothetical educational situations determined by the learners' initial level. If students are assumed as the current educational situation, the model of the educational process can be developed on the basis of the fuzzy-set theory and its practical implementation by means of computer science. Thus, the main characteristics of the intelligent learning environment are interactivity, constructive feedback, and the focus on an individual. These requirements can be met by applying specialized software that simulates real-life situations in which learners are supposed to solve problems similar to the ones they face in their professional careers.

\subsection{Literature review}

According to the authors [1], new technologies lead to the adoption of a variety of smart solutions in university environments to enhance the standard of living and to improve both teachers' and students' performance. The authors propose and discuss a smarter university model, they distinguish issues and challenges in smart education such as smart technologies, smart competencies, and smart processes, integration of which constitutes a smart educational ecosystem. Unfortunately, no technical decisions are offered in the paper.

Smart education, a concept that describes learning in the digital age, has gained increased attention and is actively investigated in many pieces of research [2]. Intelligent technologies,

\footnotetext{
* Corresponding author: kobicheva92@gmail.com
} 
such as cloud computing, learning analytics, big data, Internet of things (IoT), wearable technology and etc., promote the emergence of smart education. Zhu, Z. et al elaborated on the research framework of smart education, the main elements of which are smart environments, smart pedagogy, and a smart learner. In the paper, they proposed a tri-tier architecture of smart computing that constituted Cloud computing, Fog computing, and Swarm computing.

The paper [3] discusses the main characteristics of smart education and smart learning environments and sustains the relevance of taking the participation of future users into account during the design process to increase knowledge of the design and the implementation of new pedagogical approaches in smart learning environments. Due to their study combining the Internet of things technology with social and behavioral analysis, an ordinary educational environment can be transformed into a smart environment that actively listens and analyses voices, conversations, movements, behavior, etc., in order to reach a conclusion about the lecturers' presentation and listeners' satisfaction. Also, the paper considered the ideas of smart learning environment design and focused on two key issues that should be worked out - user participation in the design and support of feedback.

Jianshan S. et al. [4] proposed the new technology that is able to formalize personality as digital twin models by observing users' posting content and liking behavior. They used a multitask learning deep neural network model to predict users' personalities through two types of data representation. 'A similar technique can be used in a smart educational environment to reveal the students' learning needs.

Gorbunov A.A. et al. developed a simulation model using the capabilities of the tool AnyLogic 7 to estimate the load on the information system used in the educational process and support the educational process planning [5]. Such a model could be introduced as a part of smart educational infrastructure contributing to the developed technological learning environment.

Methods of organizing the intelligent learning environment have been studied by a number of researchers, such as [6-8], and many others.

\section{Innovative learning models}

Nowadays, apart from classical university education, both students and teachers face the need to adapt to the digital learning environment. Internet technologies along with the everexpanding possibilities to present knowledge in a digital form can be regarded as an additional educational means.

The Fourth Industrial revolution will boost the demand for well-qualified engineers. To this end, innovative educational technology should be deployed. At research universities, robots cannot serve as an adequate substitute for human teachers since the participants of the educational process still need live communication, teachers' advice, and team discussions. Truth is born of arguments, reflection, and a creative atmosphere [9]. Since the existing technologies improve the standards of living, they should be regarded as an inherent part of technological systems [10]. Apart from being a user of technologies, an individual is exposed to various technical activities and technical artifacts. Therefore, simulation technologies should go beyond technical artifacts and take into consideration human roles and activities related to the artifacts. When digital twins take on a human role, they are referred to as human digital counterparts (digital twins of physical processes) [11]. 
The main issues of human-technological interaction that should be addressed can be considered as an ontology illustrating how human-technology interaction concepts can be presented [12]. Ontologies usually describe the attributes of the main concepts and systems. It is essential to mention that users' ideas about the system can differ from those of design engineers and managers because they see the system from different perspectives. Anecdotal evidence suggests that complex and dynamic production processes cannot be reduced just to physics or engineering.

In fact, the digital university educational system is a combination of learning courses with a comprehensive, coherent, and structured educational content; a description of each subject area in the form of texts, images, graphs, charts along with appropriate audio- and video effects for illustration [13]. In this system, the learner, studying the subject piece by piece in accordance with the instructions given by the system, plays the leading role. Basically, modeling the educational process can be divided into three main parts: the teacher's model, the learner's model, and the area in which they interact, for example, a coursebook with exercises $[14,15]$. The educational course consists of the following components: learning materials exercises aimed at improving the knowledge and mastering the skills, the description of the learning course including the educational goals and reference information presented as a table, a glossary, etc. [16, 17].

The learner's interface usually includes the following components: the content of the learning course, the exercises included in the assignment, the access to reference and any other additional information provided by the author of the course, a learner's electronic portfolio which includes the results of assignments and recommendations given by the expert system. It also contains the learning statistics, such as both completed and unfinished exercises, mistakes made by the student, and advice given by the expert system.

An authoring system is a tool for creating, developing, and editing all parts of a learning course and giving students access to its different parts.

As is well known, the best way to educate researchers is by involving them in studies conducted by a particular scientific community. A baccalaureate does not suffice to prepare a fully-fledged researcher, so it makes sense to consider a bachelor course and a master's degree program as the two phases of the unified educational process.

The first step towards educating a researcher involves identifying the scientific communities working on the global scale within a particular university; commercially successful cutting-edge high-tech projects related to Industry 4.0 as well as individual researchers or research groups that can evolve into established scientific communities before long. Later on, future engineers-researchers should participate in the studies conducted in one of the above-mentioned scientific communities. A master's degree program or a course within a master's degree program should be assigned to each scientific community.

The whole period of baccalaureate and a master's degree program is divided into two 3 year stages. In the first stage, students are supposed to become knowledgeable about the basic subjects, and in the second three phases, they should work closely within the scientific area of their choice.

University entrants will be allowed to choose a particular scientific community. It will give the following advantages:

- It will be possible to advertise scientific communities to prospective university students instead of advertising departments or courses, which will be more appealing to enrollees, especially to the ones with higher education attainment.

- It will give an opportunity to put scientific communities in perspective, and the latter will be able to evaluate themselves. 
- Freshmen, who will be regarded as future colleagues and assistants, will be patronized by senior students belonging to the same scientific community.

In the first-year students will build up their capacity in basic subjects, which should be taught in a way comprehensible to most of the freshmen. The first-year students who have chosen to join a particular scientific community will be given entry-level individual assignments covering a certain topic. When the freshmen do these assignments, they will receive help from the senior students from the same scientific community, who, in their turn, will brush up on their knowledge of the subjects they studied in their earlier years at university and develop their leadership and interpersonal skills.

The students capable of doing a master's and who can be trained to be researchers will be selected following the results of their academic performance in the freshman year. In the course of further studies, the students can move to another scientific community by mutual consent. In the sophomore and junior years, the students will be taught the fundamentals of the subjects related to their future professional occupation. This professional training is supposed to conform to international standards. At this stage, interdisciplinary projects should play a vital role. A representative of a particular scientific community sets the goal of the project and supervises its implementation, whereas the teachers of mathematics, physics, and computer science help to complete the project by providing counselling in their field.

In the senior year, these students are given specialized training by doing elective courses aimed at the corresponding mater's program. Following the results of this course (primarily their bachelor's graduate qualification work), it will be possible to judge whether each of these students should continue their studies at the master's level. When students move on to their senior year, they can be allowed to transfer to another scientific community.

In grad school, the prospective researchers should be actively involved in creating intellectual products for their scientific community by writing articles and participating in scientific conferences and projects. It is only natural that a master's thesis should account for as much as $50 \%$ of the future Ph.D. thesis. In this case, working for an external employer along with doing a master's does not make sense.

The students who were not selected for research training at the end of their freshman year, do a professionally oriented bachelor course. They are supposed to get thorough training so that they can be in demand on the job market and pursue their professional career when they graduate.

In our previous articles, we came up with some other ideas for organizing an intelligent learning environment. In one of them, we did further research into the hierarchical teaching systems and mentorship, in which the knowledge is transferred not only from a teacher to learners but also from one student to another. We applied such mathematical models as the system of Kolmogorov differential equations for the Markovian process with a discrete set of states to test the efficiency of the systems. The socio dynamic model of the learning process developed in the course of this research enables educators to forecast and maximize its further results [18].

We also proved that hierarchical learning systems are more efficient than conventional ones by conducting computational experiments, in which real-world hierarchical teaching was simulated by hierarchical neural networks. In our research, we used the neural network approach combined with the Kolmogorov differential equation system for the Markov process and the Fokker-Planck equation. The practical upshot of this experiment was the conclusion that integrating the hierarchical approach into teaching different subjects makes sense, especially in situations with the shortage of time [19]. 
The other method considered in our previous research was a new methodology of measuring the students' level of knowledge on the basis of other students' subjective opinions. We did research into the correlation between the subjective and objective indicators of the educational

process. The method allows the teacher to evaluate the progress made by a group of learners in a less time-consuming way and to manage all the aspects of the educational process, such as the pace of learning, the needed checkpoints, the complexity level of the tests, etc. We analyzed the results of the students' mutual assessment by using the method of least squares through neural network function. [20]. Below we will suggest some further methods of simulating the learning environment.

\section{Methods of simulating the learning environment}

Some scientists suggest using a linear dynamic system with discrete time as a mathematical model of the learning process outcomes, whereas other authors suggest using non-linear polynomial regression. In our opinion, other models are more efficient.

In [21-22] we suggest using the system of Kolmogorov differential equations for the Markovian process with a discrete set of states as the main type of social system models.

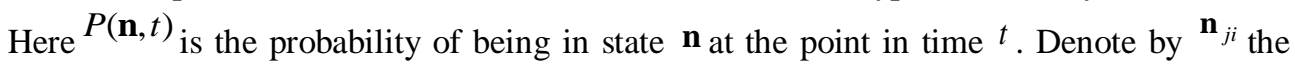
vector which is different from $\mathbf{n}$ by an increase in coordinate $j$ and a decrease in coordinate $i$ by $1 ; \mathbf{n}_{i+}$ is different by the increase in coordinate $i$ by 1 , and $\mathbf{n}_{i-}$ is different by the decrease in coordinate ${ }^{i}$ by 1 . Then the "main equation is the following:

$$
\begin{aligned}
& \frac{d P(\mathbf{n}, t)}{d t}=\sum_{i \neq j} w_{i j}\left(\mathbf{n}_{j i}, t\right) P\left(\mathbf{n}_{j i}, t\right)-\sum_{i \neq j} w_{j i}(\mathbf{n}, t) P(\mathbf{n}, t)+\sum_{i} w_{i+}\left(\mathbf{n}_{i-}, t\right) P\left(\mathbf{n}_{i-}, t\right)- \\
& -\sum_{i} w_{i+}(\mathbf{n}, t) P(\mathbf{n}, t)+\sum_{i} w_{i-}\left(\mathbf{n}_{i+}, t\right) P\left(\mathbf{n}_{i+}, t\right)-\sum_{i} w_{i-}(\mathbf{n}, t) P(\mathbf{n}, t) .
\end{aligned}
$$

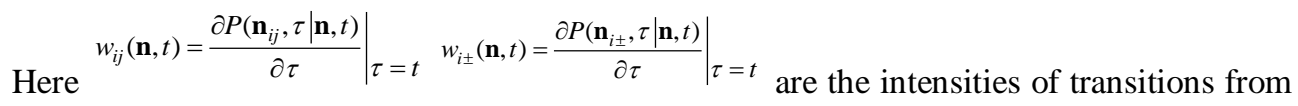
this state into the state with the increased or decreased coordinate ${ }^{i}$.

If there are many states, it is difficult to use equation (1) for the analysis and forecasting the dynamic of the real system. In book [22], in which the ideas of book [21] are developed, it was suggested that the processes with a continuous set of states should be considered. Such processes can be regarded as an approximation to the processes with a discrete set of states in the situations with a considerable number of states. Conditional density function $f(\mathbf{y}, \tau \mid \mathbf{x}, t)$ for such processes corresponds to the system of Kolmogorov differential equations:

$$
Y(h v)=\frac{1}{q} \frac{(h v)^{2}}{\left[\left(h v_{r}\right)^{2}-(h v)^{2}\right]^{2}+\left(\hbar \Delta \omega_{1 / 2}\right)^{2}(h v)^{2}} \int_{E \tau-E_{V}-\Delta \varphi}^{\infty} \frac{\left[E+\left(E_{v}-h v\right)\right]^{1 / 2}}{\left[E+\left(E_{v}-E_{-}\right)\right]^{1 / 2}} \frac{E}{\exp \left[\left(E-E_{m}\right) / k T\right]+1} \mathrm{~d} E
$$


When such models are used for forecasting, the problem of identifying functions $a$ and $b$ by real data should be addressed. It is necessary to know the initial and boundary conditions to solve these equations using the conventional methods. However, in reality, the results of the observations of the process are known. Therefore, the classical methods of solving the problems in mathematical physics, such as a net method, a finite element method, and others cannot be applied for solving (2). The methods described in works [23-31] allow building approximate solutions (2) in the neural networks [32], with regard to all the available information.

It should be mentioned that the methods and algorithms of building stable neural network models by heterogeneous data $[25,28]$ involve not only selecting the free parameters, but also the structure of the model. It is essential for the models of the educational process since the object that is supposed to be simulated is very complex.

A neural network is determined by the formation rule (a type of a network); the number of components and the way they are linked with each other (a structure of a network), and the coefficients which determine a particular function (weights of a network). A number of theorems have been proved that provide the evidence that the networks with some particular architectures are universal approximators. It means that they can arbitrarily closely approximate the function from a certain functional space, for example, a continuous function [32]. In the book [25] it is suggested combining the evolutionary algorithm of selecting the network structure and the algorithm of non-linear optimization for selecting its weights.

\section{Conclusion}

We considered introducing a hierarchical educational system as a way of training a prospective researcher. We also described some examples of using artificial intelligence (neural networks in particular) for organizing the intelligent learning environment. The results of the experiments involving neural networks can be applied in the educational process in order to achieve better learning outcomes. The intelligent learning environment can help formalize the educational process by making it more uniform and consistent. On the one hand, it can encourage teamwork and mentorship, on the other hand, students will be able to follow their individual educational trajectory depending on their basic knowledge, the pace of learning, scientific interests, and psychological characteristics. Further interdisciplinary research should be done into all aspects of the intelligent learning environment and its various applications at all educational levels.

\section{References}

1. Coccoli M, Guercio A Maresca P Stanganelli L 2018 J Visual Lang. Comput. 251003 doi: 10.1016/j.jvlc.2014.09.007

2. Zhu Z, Yu M and Riezebos P 2016 Smart Learn. Environ. 34 doi:10.1186/s40561-0160026-2

3. Shoikova E, Nikolov R, Kovatcheva E 2017 Conceptualising of smart education "E+E" 3-4 29

4. Jianshan S, Zhiqiang T, Yelin F, Jie G and Chunli L 2020 International Journal of Computer Integrated Manufacturing doi:10.1080/0951192X.2020.1757155 
5. Gorbunov A A, Isaev E A, Morgunov A F 2017 Business Informatics 2(40) 57 doi: 10.17323/1998-0663.2017.2.57.67

6. Aladyshkin I V, Kulik S V, Odinokaya M A, Safonova A S and Kalmykova S V 2020 Proceedings of the Conference "Integrating Engineering Education and Humanities for Global Intercultural Perspectives" (Springer Cham) p 659

7. Barinova D, Ipatov O, Odinokaya M and Zhigadlo V 2019 Annals of DAAAM \& Proceedings 30

8. Andreeva A, Mikhailova O, Petrov M, Odinokaya M and Pyatnitsky N 2020 E3SWC 16412011

9. Sitnicki M 2018 Baltic Journal of Economic Studies 4(1) 311

10. Saariluoma P, Cañas J and Karvonen A 2020 International Conference on Human Interaction and Emerging Technologies (Springer Cham) p 97

11. Wilson H J and Daugherty P R 2018 Harvard Business Review 96(4) 114

12. Saariluoma $P$ and Leikas $J 2020$ International Conference on Intelligent Human Systems Integration (Springer Cham) 594

13. Kudrjavcev V, Waschik K, Strogalov, A, Alisejtshik P and Peretruchin V 1996 Modeling educational process using expert system New Media for Education and Training in Computer Science p 89

14. Krepkaia T, Karpovich I, Odinokaya M, Ivanova T 2019 Educ. Sci. 9200

15. Bernavskaya M, Odinokaya M, Krepkaia T, Sheredekina O 2019 Educ. Sci. 9187

16. Almazova N, Bernavskaya M, Barinova D, Odinokaya M and Rubtsova A 2020 Proceedings of the Conference "Integrating Engineering Education and Humanities for Global Intercultural Perspectives" (Springer Cham) 786

17. Baranova T A, Kobicheva A M, Tokareva E Y 2019 St. Petersburg Polytechnic University ACM International Conference Proceeding Series p 77 doi.org/10.1145/3369199.3369245

18. Tarkhov D A, Dashkina A I 2017 Complex Systems Theory and applications (NY: Nova Publishers) p 95

19. Tarkhov D A, Blagoveshchenskaya E A, Lazovskaya T V, Ryabukhina V V 2016 Advances in Neural Networks 513

20. Zulkarnay I U, Tarkhov D A, Dashkina A I 2018 Journal of Physics: Conference Series Measurement Science Challenges in Natural and Social Sciences Symposium: Measurement Science Challenges in Natural and Social Sciences

21. Weidlich W, 2000 Sociodynamics A systematic approach to mathematical modelling in the social sciences (Amsterdam: Harwood Academic) p 380

22. Helbling D 2010 Quantative Sociodynamics Heidelberg Dordrecht (London, New York: Springer) p 333

23. Lagaris I E, Likas A, Fotiadis D I 1998 IEEE Transactions on Neural Networks 9(5) 987

24. Rudd K, Ferrari S 2015 Neurocomputing 155277

25. Dorman L I 1975 Variations of Galactic Cosmic Rays (Moscow: Moscow State University Press) p 103 
26. Tarkhov D, Vasilyev A 2019 Semi-empirical Neural Network Modeling and Digital Twins Development Academic (Press Elsevier) p 288 https://www.elsevier.com/books/semi-empirical-neural-network-modeling-and-digitaltwins-development/tarkhov/978-0-12-815651-3

27. Tarkhov D A, Vasilyev A N 2005 Allerton Press Inc. 14(1) 59

28. Tarkhov D A, Vasilyev A N 2005 Allerton Press Inc. 14(2) 97

29. Antonov V, Tarkhov D, Vasilyev A 2018 Applied Sciences 1-8 do:10.1002/mma.5205

30. Budkina E M, Kuznetsov E B, Lazovskaya T V, Tarkhov D A, Shemyakina T A, Vasilyev A N 2017 Optical Memory and Neural Networks 26(2) 96 https://link.springer.com/article/10.3103/S1060992X17020011

31. Vasilyev A N, Tarkhov D A 2014 Complex Systems 17(3) 327

32. Lazovskaya T N, Tarkhov D A, Vasilyev A N 2017 Engineering Recent Patents on Engineering 11(1) 10

33. Haykin S 1999 Neural Networks: A Comprehensive Foundation (Prentice Hall) p 823 